\title{
Streptococcus mitis Abscesses Mimicking Liver Metastases
}

\author{
Mohamed Elnaggar $^{1}$, Sumaiya Mahboob ${ }^{1}$, Bryce D. Beutler ${ }^{1}$, Ahmed Hanfy ${ }^{1}$, Omar Canaday ${ }^{1}$ \\ 1. Internal Medicine, University of Nevada, Reno School of Medicine, Reno, USA
}

Corresponding author: Bryce D. Beutler, brycebeutler@hotmail.com

\begin{abstract}
Streptococcus mitis (S. mitis) is a commensal bacterial species that commonly colonizes the oropharynx and gastrointestinal tract. It is seldom reported as a human pathogen. However, immunocompromised individuals may be at risk of infection. We describe a 62 -year-old male with prostate cancer who presented with multiple S. mitis abscesses masquerading as metastases. In addition, we discuss the differential diagnosis and treatment options for this rare opportunistic infection.
\end{abstract}

Categories: Infectious Disease, Oncology

Keywords: abscesses, liver metastases, pyogenic abscess, streptococcus mitis

\section{Introduction}

Streptococcus mitis (S. mitis, previously known as Streptococcus mitior) is a mesophilic, facultatively anaerobic, gram-positive coccus. A member of the viridans group streptococci (VGS), S. mitis is most commonly found in the throat, nasopharynx, and oral cavity. It usually considered to have low virulence and pathogenicity. However, S. mitis endocarditis and meningitis have been reported $[1,2]$.

In the United States, liver abscesses are most commonly polymicrobial and attributed to Escherichia coli and Klebsiella pneumoniae [3]. Entamoeba histolytica and Candida species account for the majority of nonbacterial abscesses $[4,5]$. We describe a patient with prostate cancer who presented with multiple liver lesions that were later revealed to be pyogenic abscesses caused by S. mitis.

\section{Case Presentation}

A 62-year-old male with stage III prostate cancer undergoing treatment with leuprolide and radiation therapy presented with a one-week history of anorexia, night sweats, subjective fevers, and abdominal pain.

Received 06/15/2020 Review began 06/23/2020 Review ended 06/24/2020 Published 06/26/2020

(c) Copyright 2020 Elnaggar et al. This is an open access article distributed under the terms of the Creative Commons Attribution License CC-BY 4.0., which permits unrestricted use, distribution, and reproduction in any medium, provided the original author and source are credited.
The patient was afebrile and hemodynamically stable in the emergency department. Physical examination was significant only for right-sided abdominal tenderness. Laboratory studies showed a mild leukocytosis (white blood cell count: $13.3 \mathrm{~K} / \mu \mathrm{L}$; reference range: $4.5-11.0 \mathrm{~K} / \mu \mathrm{L}$ ) with bandemia, anemia (hemoglobin: 8.5 $\mathrm{g} / \mathrm{dL}$; reference range [male]: 14.0-18.0 g/dL), and elevated alkaline phosphatase (alkaline phosphatase: 276 $\mathrm{U} / \mathrm{L}$; reference range: $20-140 \mathrm{U} / \mathrm{L}$ ). Prostate-specific antigen was $<0.01 \mathrm{ng} / \mathrm{mL}$ (reference range: 0.0-4.0 $\mathrm{ng} / \mathrm{mL}$ ). Bilirubin, alanine aminotransferase, and aspartate aminotransferase were within normal limits. Renal function studies were unremarkable. Urinalysis was negative for leukocyte esterase and nitrites. Correlation of the clinical history and physical examination findings was worrisome for an occult infection, prompting emergency department providers to obtain additional imaging.

An abdominal ultrasound demonstrated several poorly demarcated hypoechoic masses affecting the liver. A CT scan of the abdomen and pelvis with contrast was obtained to further characterize the lesions; this showed numerous peripherally enhancing, centrally hypoattenuating masses in liver segments II, III, and IV (Figure 1). All lesions were new as compared to a CT scan from two months earlier. 


\section{Cureus}

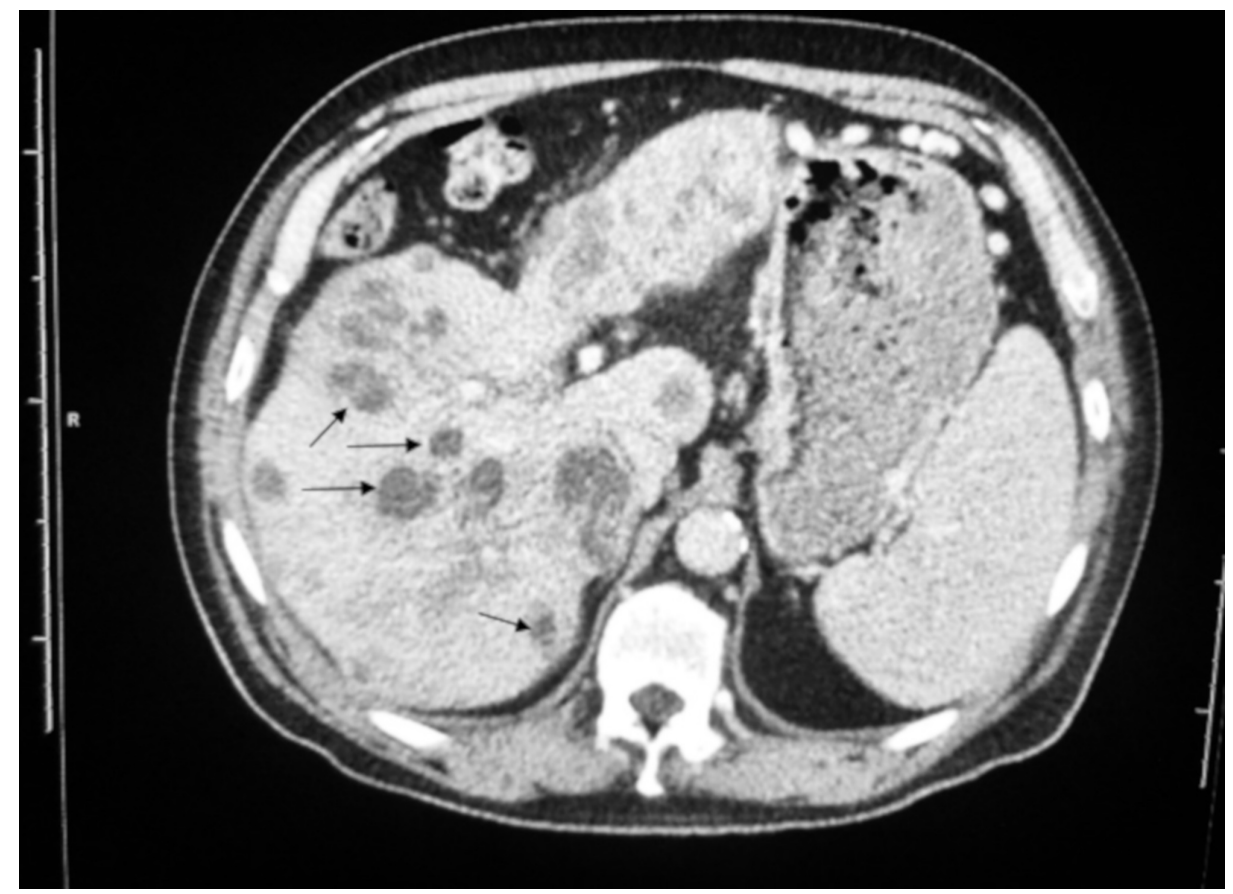

FIGURE 1: Axial view CT scan of the abdomen and pelvis with contrast shows numerous peripherally enhancing, centrally hypoattenuating masses in liver segments II, III, and IV.

The differential diagnosis included liver abscesses and cystic metastases. Blood, sputum, stool, and urine cultures were acquired, and the patient was started on intravenous ceftriaxone and metronidazole. A final needle biopsy of a lesion was obtained for pathologic examination.

Tissue cultures grew S. mitis. Infectious disease was consulted and recommended intravenous ceftriaxone and metronidazole. The patient's symptoms gradually began to improve, and he was discharged in a stable condition after a two-week hospital stay. Daily infusions of intravenous ceftriaxone was continued on an outpatient basis for a duration of six weeks. The patient continued to follow up with the infectious disease clinic. Repeat CT scan of the abdomen and pelvis with contrast obtained one month after completion of antibiotic therapy revealed resolution of the abscesses (Figure 2). 


\section{Cureus}

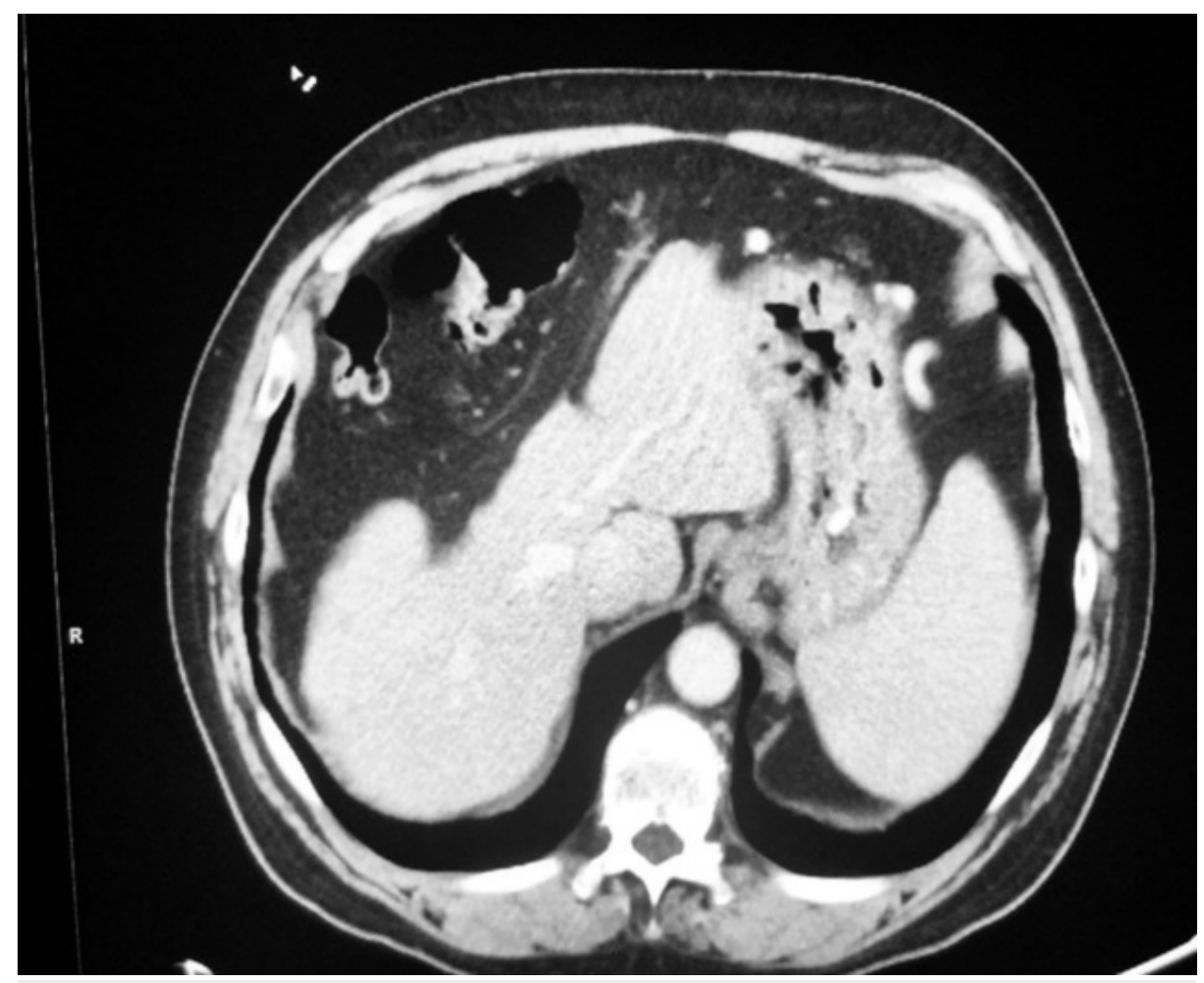

FIGURE 2: Axial view of a CT scan of the abdomen and pelvis with contrast demonstrates complete resolution of the previously observed Streptococcus mitis abscesses.

\section{Discussion}

S. mitis is a rare but important opportunistic pathogen. In the setting of cancer, S. mitis abscesses may appear similar to metastases on imaging. Therefore, careful correlation of clinical history, physical examination, and laboratory and imaging findings is essential in order to establish a diagnosis. In our patient, pyogenic abscesses were favored over metastases for the following reasons: (1) the subacute onset of subjective fevers and abdominal pain is consistent with infection; (2) the liver represents an uncommon site for prostate cancer metastases; (4) prostate-specific antigen remained within normal limits; and (4) imaging studies obtained two months prior to presentation revealed no hepatic lesions.

S. mitis is a commensal organism rarely known to cause infection. To the best of our knowledge, only two other cases of S. mitis abscesses have been described in the medical literature [6,7]. Our patient was unique in that he had no history of immunosuppression. Previous studies of S. mitis bacteremia have shown that this opportunistic pathogen is usually susceptible to ceftriaxone; tetracycline represents an alternative therapy for individuals who are unable to tolerate cephalosporins [8]. In the setting of S. mitis abscesses, prolonged therapy may be required.

\section{Conclusions}

Pyogenic abscesses are typically polymicrobial. However, albeit rarely, healthy patients may be susceptible to infection with atypical organisms, including S. mitis. The imaging findings of S. mitis abscesses are virtually indistinguishable from those of metastases. Careful clinical correlation is therefore required to establish a diagnosis and administer the appropriate treatment.

\section{Additional Information}

\section{Disclosures}

Human subjects: Consent was obtained by all participants in this study. Conflicts of interest: In compliance with the ICMJE uniform disclosure form, all authors declare the following: Payment/services info: All authors have declared that no financial support was received from any organization for the submitted work. Financial relationships: All authors have declared that they have no financial relationships at present or within the previous three years with any organizations that might have an interest in the submitted work. Other relationships: All authors have declared that there are no other relationships or activities that could appear to have influenced the submitted work. 


\section{Cureus}

\section{References}

1. Lamas CC, Eykyn SJ: Blood culture negative endocarditis: analysis of 63 cases presenting over 25 years . Heart. 2003, 89:258-262. 10.1136/heart.89.3.258

2. Kutlu SS, Sacar S, Cevahir N, Turgut H: Community-acquired Streptococcus mitis meningitis: a case report . Int J Infect Dis. 2008, 12:107-109. 10.1016/j.ijid.2008.01.003

3. Johannsen EC, Sifri CD, Madoff LC: Pyogenic liver abscesses. Infect Dis Clin North Am. 2000, 14:547-563. 10.1016/s0891-5520(05)70120-3

4. Meddings L, Myers RP, Hubbard J, et al.: A population-based study of pyogenic liver abscesses in the United States: incidence, mortality, and temporal trends. Am J Gastroenterol. 2010, 105:117-124.

10.1038/ajg.2009.614

5. Kurland JE, Brann OS: Pyogenic and amebic liver abscesses. Curr Gastroenterol Rep. 2004, 6:273-279. 10.1007/s11894-004-0078-2

6. Shea KW, Schoch PE, Klein NC, Cunha BA: Liver abscess due to pyridoxal-dependent Streptococcus mitis . Clin Infect Dis. 1995, 21:238-239. 10.1093/clinids/21.1.238

7. Sarthy J, DiBardino D: Pyogenic liver abscesses caused by Streptococcus mitis . Lancet Infect Dis. 2013, 13:822. 10.1016/S1473-3099(13)70166-X

8. Shelburne SA, Shahasrabhojane P, Saldana M, et al.: Streptococcus mitis strains causing severe clinical disease in cancer patients. Emerg Infect Dis. 2014, 20:762-771. 10.3201/eid2005.130953 